\title{
Optimization of structures in frictional contact
}

\author{
Daniel Hilding and Anders Klarbring
}

\section{Linköping University Post Print}

N.B.: When citing this work, cite the original article.

Original Publication:

Daniel Hilding and Anders Klarbring, Optimization of structures in frictional contact, 2012, Computer Methods in Applied Mechanics and Engineering, (205-208), S1, 83-90.

http://dx.doi.org/10.1016/j.cma.2011.02.014

Copyright: Elsevier

http://www.elsevier.com/

Postprint available at: Linköping University Electronic Press

http://urn.kb.se/resolve?urn=urn:nbn:se:liu:diva-73555 


\title{
Optimization of structures in frictional contact
}

\author{
Daniel Hilding and Anders Klarbring \\ Division of Mechanics \\ Institute of Technology, Linköping University \\ SE-581 83 Linköping, Sweden \\ anders.klarbring@liu.se
}

\begin{abstract}
This paper describes a new approach to optimization of linear elastic structures in frictional contact. It uses a novel method to determine an, in a specified sense, likely equilibrium state of the structure, using only the static equilibrium conditions. That is, no complex dynamic/quasi-static analyses have to be performed. The approach has the advantage that it is not necessary to know the complete load history, which is most often unknown for practical problems. To illustrate the theory, numerical results are given for the optimal design problem of sizing a truss to attain a more uniform normal contact force distribution.
\end{abstract}

Keywords: contact, friction, structural optimization, sensitivity analysis

\section{Introduction}

Consider finding the optimal design of a connecting element transmitting torque and force by means of frictional forces (say, a press-fitted gear wheel). The treatment of such a design problem by means of mathematical optimization requires that we know the state (for each conceivable design) of the connecting element in terms of contact forces and displacements. A major concern then arises: the state of a linear elastic body in frictional contact depends on the load history and not only on the momentary value of the load, and such a load history is in practice not known in detail or even in an approximate sense. A remedy of this situation was suggested in Hilding (2000) (based on an idea in Klarbring and Pang (1996)). In 
that paper the elastic frictional contact problem was relaxed by simply removing the condition involving time derivatives (specifying the direction of frictional slip) and, thus, making the concern about load histories disappear. Obviously, contact forces and displacements satisfying the remaining equations will be (highly) nonunique, but Hilding then introduced the idea of sorting among these solutions by picking those that give the lowest value for the potential energy (and which satisfies a certain additional constraint concerning rigid body modes). These minimum potential energy solutions were called likely states, and it was shown that if the corresponding frictionless contact problem has a unique solution, a likely state is also unique and coincides with the frictionless solution. However, by the presence of friction forces, the so formulated likely-state problem has solutions also when the frictionless problem has not. Therefore, this problem can be considered a natural static generalization of the frictionless problem to the many situations where frictional forces are necessary for satisfying equilibrium.

In the present paper we consider optimal design of structures required to be in a likely state, thus, effectively indicating a new method for optimal design of friction based connecting elements. We discuss sensitivity analysis in a general setting where the design variable may effect the stiffness matrix as well as the contact gap vector. However, the explicit optimization problem formulation is for sizing of trusses where cross-sectional areas of bars are design variables, but it is straightforward to apply the approach also to finite element modeled structures.

Optimization of structures in frictional contact has been attempted in a number of previous papers, e.g., Rodrigues (1993), Stavroulakis (1995), Kočvara and Outrata (1997), and Outrata et al. (1998). However, these are based on the static friction problem of Duvaut and Lions (1976) and since this model only makes physical sense when considered as a step in a time sequence of incremental problems, produced by discretizing the quasistatic friction problem, we find these approaches highly questionable. In fact, the same critique holds also with respect to some older work of the second author of this paper that was based on a modification of the Duvaut and Lions (1976) model, see Klarbring et al. (1992a) and Klarbring et al. (1992b), as well as Haslinger and Neittaanmaki (1996).

The paper is organized as follows. In Sec. 2 the static equilibrium conditions for a truss structure are given together with the likely-state problem. Expressions for the sensitivities of the likely-state problem with respect to the design variable are derived in Sec. 3. The sensitivities are necessary as a sequential programming algorithm is used to solve the structural optimization problem. In Sec. 4 the structural optimization problem used as test problem is formulated. It is the problem of sizing a truss to minimize the maximum normal contact force. Techniques for solving this structural optimization problem are discussed in Sec. 5. In particular, we chose to 
use the method of moving asymptotes (MMA), see Svanberg (1987), which is a widely used method in structural optimization. Finally, in Sec. 6 the developed theory is demonstrated on a few example structures.

\section{Formulation of the likely-state problem}

In this section we formulate the likely-state problem for a given design of a truss structure. A thorough derivation and analysis of this problem can be found in Hilding (2000).

\subsection{Static equilibrium conditions}

Consider the three-dimensional truss structure in Figure 1. The structure is assumed linear elastic and small deformations are assumed. A number $n_{c}$ of the nodes in the truss may come into frictional contact with one of $n_{c}$ plane rigid obstacles. The state of the structure is described by a

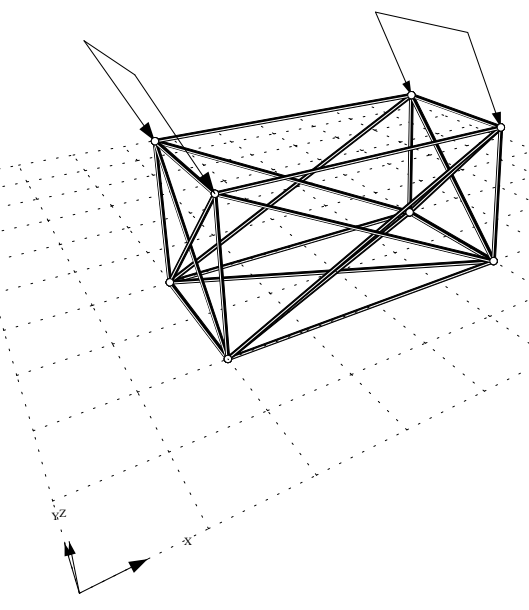

Figure 1: A truss in frictional contact with a plane rigid obstacle.

vector $\boldsymbol{u} \in \mathbb{R}^{n_{u}}$ containing the displacements of the structure and vectors $\boldsymbol{p}_{n}=\left(p_{n, i}\right), \boldsymbol{p}_{o}=\left(p_{o, i}\right)$ and $\boldsymbol{p}_{t}=\left(p_{t, i}\right)$ containing the contact forces. For obstacle $i, p_{n, i}$ is the component of the contact force that is normal to the obstacle and $p_{o, i}$ and $p_{t, i}$ are mutually orthogonal tangential components of this force. The external forces on the structure are collected in a vector $f \in \mathbb{R}^{n_{u}}$ that can be additively decomposed into contact forces $\boldsymbol{f}^{c}$ and other forces $f^{o}$. The contact forces $f^{c}$ can be expressed in terms of its components:

$$
\boldsymbol{f}^{c}=-\boldsymbol{C}_{n}^{T} \boldsymbol{p}_{n}-\boldsymbol{C}_{o}^{T} \boldsymbol{p}_{o}-\boldsymbol{C}_{t}^{T} \boldsymbol{p}_{t}
$$


Here $\boldsymbol{C}_{n}, \boldsymbol{C}_{o}$, and $\boldsymbol{C}_{t}$ are force transformation matrices containing unit vectors representing the obstacle. Hence, if the positive semidefinite stiffness matrix of the structure is denoted by $\boldsymbol{K}$, the force equilibrium of the linear elastic structure can be written as

$$
\boldsymbol{K} \boldsymbol{u}=\boldsymbol{f}=\boldsymbol{f}^{o}-\boldsymbol{C}_{n}^{T} \boldsymbol{p}_{n}-\boldsymbol{C}_{o}^{T} \boldsymbol{p}_{o}-\boldsymbol{C}_{t}^{T} \boldsymbol{p}_{t} .
$$

The fact that a node in the truss cannot penetrate an obstacle is expressed by Signorini's unilateral contact law

$$
\begin{aligned}
\boldsymbol{n}_{i}^{T} \boldsymbol{u} \leq g_{i}, i=1 \ldots n_{c} & \Leftrightarrow \boldsymbol{C}_{n} \boldsymbol{u} \leq \boldsymbol{g}, \\
p_{n, i} \geq 0, i=1 \ldots n_{c} & \Leftrightarrow \boldsymbol{p}_{n} \geq \mathbf{0}, \\
p_{n, i}\left(\boldsymbol{n}_{i}^{T} \boldsymbol{u}-g_{i}\right)=0, i=1 \ldots n_{c} & \Leftrightarrow \boldsymbol{p}_{n} \odot\left(\boldsymbol{C}_{n} \boldsymbol{u}-\boldsymbol{g}\right)=\mathbf{0},
\end{aligned}
$$

where $g_{i}$ is the gap between the obstacle and the node in the undeformed configuration. The vector $\boldsymbol{n}_{i}$ is the normal vector of the obstacle and $\boldsymbol{n}_{i}^{T} \boldsymbol{u}$ is the movement of the node towards the obstacle when the truss undergoes a displacement $\boldsymbol{u}$.

The friction condition used is that the contact forces should belong to the so-called Coulomb friction cone. This is written as

$$
\sqrt{p_{o, i}^{2}+p_{t, i}^{2}} \leq \mu_{i} p_{n, i}, i=1 \ldots n_{c} \Leftrightarrow\left(\boldsymbol{p}_{o}, \boldsymbol{p}_{t}\right) \in \mathcal{F}\left(\boldsymbol{p}_{n}, \boldsymbol{\mu}\right),
$$

where $\mu_{i} \geq 0$ is the coefficient of friction and $\mathcal{F}$ is a set-valued function.

Taken together (2), (3-5), and (6) constitute conditions for the static equilibrium of the truss. In contrary to the frictionless case, when $\left(\boldsymbol{p}_{o}, \boldsymbol{p}_{t}\right)=$ $\mathbf{0}$, there are generally many displacements and contact forces that satisfy these equations for given data. In the following subsection we give a condition that reduces the size of this set considerably.

\subsection{Likely state of a truss subject to static loads}

A likely state is defined as the state satisfying the static equilibrium conditions with the smallest potential energy. This is in close analogy to the frictionless contact problem, where the equilibrium state is the state with smallest potential energy that satisfies the contact constraints. The likelystate problem is meant to be an extension of the frictionless contact problem to structures that can perform rigid body motions and where frictional forces are necessary to satisfy the equilibrium conditions, see the structure in Figure 2 for an example. It may be shown that for structures where friction is not necessary to satisfy the equilibrium conditions the likely-state problem reduces to the frictionless contact problem, see Hilding (2000).

The potential energy of the structure is defined as

$$
\Pi(\boldsymbol{u})=\frac{1}{2} \boldsymbol{u}^{T} \boldsymbol{K} \boldsymbol{u}-\boldsymbol{u}^{T} \boldsymbol{f}^{o}
$$




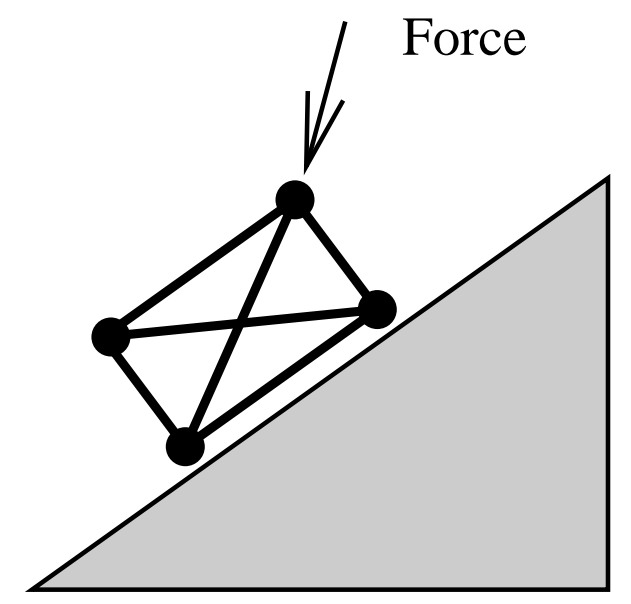

Figure 2: A small truss on an inclined plane; a structure where frictional contact is necessary for satisfying the equilibrium conditions.

where the work of the non-conservative frictional forces is not included. A likely state is defined as a solution of the following optimization problem, which is referred to as the likely-state problem:

$$
\begin{aligned}
& \min _{\boldsymbol{u}, \boldsymbol{p}_{n}, \boldsymbol{p}_{o}, \boldsymbol{p}_{t}} \Pi(\boldsymbol{u}) \\
& \text { subject to (2), (3-5), (6) and } \boldsymbol{v}_{i}^{T} \boldsymbol{u}=0, i=1 \ldots m \text {. }
\end{aligned}
$$

The constraints $\boldsymbol{v}_{i}^{T} \boldsymbol{u}=0, i=1 \ldots m$ limit the rigid body motion(s) of the structure. The vector $\boldsymbol{v}_{i}$ is the eigenvector of the rigid body motion to be limited. That it may be necessary to limit the rigid body motions is apparent from the structure in Figure 2. By moving the truss down the incline (a rigid body motion) the potential energy may be decreased indefinitely. Hence, if this rigid body motion is not limited, then (8) will not have a solution for this problem regardless of the size of the coefficient of friction.

Selecting the number of and which rigid body motions that need to be limited for (8) to have a solution can often be done by inspection. The following, unproven, rule seems also to work: let $\left\{\boldsymbol{v}_{i}\right\}$ be the smallest set of $m$ linearly independent vectors of the null-space of $\boldsymbol{K}$ such that the static equilibrium conditions (2), (3-5), (6) with $\boldsymbol{K}$ replaced by $\boldsymbol{K}+\sum_{i=1}^{m} \boldsymbol{v}_{i} \boldsymbol{v}_{i}^{T}$ has a solution with $\boldsymbol{p}_{o}$ and $\boldsymbol{p}_{t}$ both equal to $\mathbf{0}$. This rule is based on the fact that the null-space of $\boldsymbol{K}$ is exactly the space of displacements corresponding to the possible rigid-body motions of the truss if there where no contact constraints. 


\section{Sensitivity analysis of the likely-state problem}

Assume that the stiffness matrix $\boldsymbol{K}$ and gap vector $\boldsymbol{g}$ in the static equilibrium conditions are functions of a design variable $s \in \mathbb{R}$. In this section the sensitivities, i.e., derivatives, of the solution of the likely-state problem (8) will be determined with respect to $s$ at a point $s^{*}$. The expressions for the sensitivities are derived by rewriting the likely-state problem into a form for which there are known results. As a blanket assumption we suppose that the solution of the likely-state problem is unique for all $s$ in some nonempty open neighborhood $S$ of $s^{*}$. The fact that the only non-convex condition of this problem is the complementarity condition (5) indicates that this may indeed be true in a large number of cases.

Denote the solution of the likely-state problem in the neighborhood $S$ by $\left(\boldsymbol{u}(s), \boldsymbol{p}_{n}(s), \boldsymbol{p}_{o}(s), \boldsymbol{p}_{t}(s)\right)$ and the solution at $s^{*}$ by $\left(\boldsymbol{u}^{*}, \boldsymbol{p}_{n}^{*}, \boldsymbol{p}_{o}^{*}, \boldsymbol{p}_{t}^{*}\right)$. Define (for $s \in S$ ) the following contact sets

$$
\begin{aligned}
I_{+}(s) & =\left\{i:\left(\boldsymbol{C}_{n} \boldsymbol{u}(s)-\boldsymbol{g}(s)\right)_{i}=0 \text { and } p_{n, i}(s)>0\right\}, \\
I_{0}(s) & =\left\{i:\left(\boldsymbol{C}_{n} \boldsymbol{u}(s)-\boldsymbol{g}(s)\right)_{i}=0 \text { and } p_{n, i}(s)=0\right\}, \\
I_{-}(s) & =\left\{i:\left(\boldsymbol{C}_{n} \boldsymbol{u}(s)-\boldsymbol{g}(s)\right)_{i}<0 \text { and } p_{n, i}(s)=0\right\},
\end{aligned}
$$

and

$$
J_{+}(s)=\left\{i: p_{n, i}>0 \text { and } \sqrt{p_{o, i}^{2}+p_{t, i}^{2}}=\mu_{i} p_{n, i}\right\} .
$$

The two following lemmas are first steps toward an expression for the sensitivities.

Lemma 3.1 Suppose that in the neighborhood $S^{\prime} \subset S$ of $s^{*}$ it holds that $I_{-}(s), I_{+}(s)$, and $J_{+}(s)$ do not depend on $s$ (they will be referred to simply as $I_{-}, I_{+}$, and $\left.J_{+}\right)$and $I_{0}(s)$ is empty, then for all $s \in S^{\prime}$ the following optimization problem has the same solution as the likely-state problem (8)

$$
\begin{aligned}
& \min _{\boldsymbol{u}, \boldsymbol{p}_{n}, \boldsymbol{p}_{o}, \boldsymbol{p}_{t}} \Pi(\boldsymbol{u}, s) \\
& \text { subject to } \boldsymbol{h}\left(\boldsymbol{u}, \boldsymbol{p}_{n}, \boldsymbol{p}_{o}, \boldsymbol{p}_{t}, s\right)=\mathbf{0}
\end{aligned}
$$

where

$$
\Pi(\boldsymbol{u}, s)=\frac{1}{2} \boldsymbol{u}^{T} \boldsymbol{K}(s) \boldsymbol{u}-\boldsymbol{u}^{T} \boldsymbol{f}^{o}
$$

and $\boldsymbol{h}: \mathbb{R}^{n u+3 n_{c}+1} \rightarrow \mathbb{R}^{n}$ is

$$
\boldsymbol{h}\left(\boldsymbol{u}, \boldsymbol{p}_{n}, \boldsymbol{p}_{o}, \boldsymbol{p}_{t}, s\right)=\left[\begin{array}{l}
\boldsymbol{K}(s) \boldsymbol{u}-\boldsymbol{f}^{o}+\boldsymbol{C}_{n}^{T} \boldsymbol{p}_{n}+\boldsymbol{C}_{o}^{T} \boldsymbol{p}_{o}+\boldsymbol{C}_{t}^{T} \boldsymbol{p}_{t} \\
{\left[\boldsymbol{v}_{i}^{T} \boldsymbol{u}\right]_{i \in[1 \ldots m]}} \\
{\left[\left(\boldsymbol{C}_{n} \boldsymbol{u}-\boldsymbol{g}(s)\right)_{i}\right]_{i \in I_{+}}} \\
{\left[p_{n, i}\right]_{i \in I_{-}}} \\
{\left[\sqrt{\left(p_{o, i}\right)^{2}+\left(p_{t, i}\right)^{2}}-\mu_{i} p_{n, i}\right]_{i \in J_{+}}} \\
{\left[p_{o, i}\right]_{i \in I_{-}}} \\
{\left[p_{t, i}\right]_{i \in I_{-}}}
\end{array}\right] .
$$


Proof. Pick an arbitrary $s \in S^{\prime}$. By assumption $\left(\boldsymbol{u}(s), \boldsymbol{p}_{n}(s), \boldsymbol{p}_{o}(s), \boldsymbol{p}_{t}(s)\right)$ is the unique solution of (8). The solution may be verified to be feasible also to (13). Further, the feasible set of (13) is a subset of that of (8). Hence, as (13) has the same cost function as (8) it holds that $\left(\boldsymbol{u}(s), \boldsymbol{p}_{n}(s), \boldsymbol{p}_{o}(s), \boldsymbol{p}_{t}(s)\right)$ is the unique solution of (13).

Let $\nabla f$ denote the gradient of a function $f$ with respect to $\left(\boldsymbol{u}, \boldsymbol{p}_{n}, \boldsymbol{p}_{o}, \boldsymbol{p}_{t}\right)$.

Lemma 3.2 The Karush-Kuhn-Tucker conditions of (13) at $\left(\boldsymbol{u}^{*}, \boldsymbol{p}_{n}^{*}, \boldsymbol{p}_{o}^{*}, \boldsymbol{p}_{t}^{*}, s^{*}\right)$ are:

$$
\nabla L\left(\boldsymbol{u}^{*}, \boldsymbol{p}_{n}^{*}, \boldsymbol{p}_{o}^{*}, \boldsymbol{p}_{t}^{*}, \boldsymbol{\lambda}^{*}, s^{*}\right)=\mathbf{0},
$$

where $\boldsymbol{\lambda}^{*} \in \mathbb{R}^{n}$ is the vector of Lagrange multipliers and $L: \mathbb{R}^{n_{u}+3 n_{c}+n+1} \rightarrow$ $\mathbb{R}$ is

$$
L\left(\boldsymbol{u}, \boldsymbol{p}_{n}, \boldsymbol{p}_{o}, \boldsymbol{p}_{t}, \boldsymbol{\lambda}, s\right)=\Pi(\boldsymbol{u}, s)+\sum_{i=1}^{n} h_{i}\left(\boldsymbol{u}, \boldsymbol{p}_{n}, \boldsymbol{p}_{o}, \boldsymbol{p}_{t}, s\right) \lambda_{i} .
$$

Proof. The proof is standard.

Using the above lemmas the sensitivities of the likely-state problem can be derived by applying standard sensitivity analysis theory for nonlinear programs to (13). Denote the Hessian matrix of $f$ with respect to $\left(\boldsymbol{u}, \boldsymbol{p}_{n}, \boldsymbol{p}_{o}, \boldsymbol{p}_{t}\right)$ by $\nabla^{2} f$. (The element of row $i$ and column $j$ of the Hessian matrix of a function $f$ with respect to $\boldsymbol{x}$ is given by $\partial^{2} f /\left(\partial x_{i} \partial x_{j}\right)$.)

Theorem 3.1 Suppose that (i) the prerequisites of Lemma 3.1 hold at $s^{*}$, (ii) the second-order sufficiency condition (Fiacco (1983)) holds for (13), (iii) the gradients $\nabla h_{i}\left(\boldsymbol{u}^{*}, \boldsymbol{p}_{n}^{*}, \boldsymbol{p}_{o}^{*}, \boldsymbol{p}_{t}^{*}, s^{*}\right)$ are linearly independent, and (iv) $\boldsymbol{K}$ and $\boldsymbol{g}$ are $C^{1}$ in a neighborhood of $s^{*}$, then the solution $\left(\boldsymbol{u}, \boldsymbol{p}_{n}, \boldsymbol{p}_{o}, \boldsymbol{p}_{t}\right)$ of the likely-state problem is differentiable at $s^{*}$ and the derivative is given by

$$
\left[\begin{array}{c}
\partial \boldsymbol{u} / \partial s \\
\partial \boldsymbol{p}_{n} / \partial s \\
\partial \boldsymbol{p}_{o} / \partial s \\
\partial \boldsymbol{p}_{t} / \partial s \\
\partial \boldsymbol{\lambda} / \partial s
\end{array}\right]=-\left[\begin{array}{c|c}
\nabla^{2} L & \nabla h_{1}, \ldots, \nabla h_{n} \\
\hline \nabla h_{1}^{T} & \\
\vdots & 0 \\
\nabla h_{n}^{T} &
\end{array}\right]^{-1} \cdot\left[\begin{array}{c}
\partial \nabla L / \partial s \\
\hline \partial h_{1} / \partial s \\
\vdots \\
\partial h_{n} / \partial s
\end{array}\right]
$$

Proof. Due to (i) there is an open non-empty neighborhood $S^{\prime}$ of $s^{*}$ where (13) (with fixed sets $I_{-}, I_{+}$, and $J_{+}$) has the same solution as (8). The sensitivities of (13) are therefore same as those of (8). Hence, it is sufficient to show that (17) are the sensitivities of (13).

Assumptions (ii-iv) are the assumption necessary for Corollary 3.2.3 in Fiacco (1983) to hold for (13) at $s^{*}$, which yields the expression (17) for the sensitivities of (13). 
To calculate the sensitivities using the method of Theorem 3.1 the vector of multipliers $\boldsymbol{\lambda}^{*}$ of (13) at $s^{*}$ is needed. Under the assumptions of Theorem 3.1 , the vector $\boldsymbol{\lambda}^{*}$ can be calculated from the system of linear equations (15). This system may be over-determined, but not under-determined, and can be solved by for instance the method of normal equations, see Golub and van Loan (1989).

\section{Structural optimization problem}

This paper uses the nested approach to structural optimization, see Christensen and Klarbring (2009), which means that the state of the structure is seen as a function of the design variable. The nested approach is known to be efficient and practical for a large variety of structural optimization problems. Several optimization methods have been developed specifically for this approach, notably the MMA method, see Svanberg (1987).

\subsection{Minimizing maximum contact force to obtain uniform contact forces}

The problem of sizing a truss to minimize the maximum normal contact force is considered. This usually implies that we obtain the most uniform normal contact force distribution. Sizing of a truss in this context means sizing the cross-section areas of the bars in the truss. In some problems (as in Experiment 1 below) global equilibrium conditions set a value on the sum of all normal contact forces (the total contact force). In some other problems (like in the press-fit situation of Experiment 2 below), on the other hand, it is natural to prescribe the minimum value of this sum. For the first class of problems there is no need for the constraint on the minimum total contact force that is present in the optimization problem below; there is however no need to remove the constraint as it can be disabled simply by setting the limit $P$ on the minimum contact force to zero. Simple design constraints in the form of an upper limit $V$ of the available amount of material and upper and lower limits on the cross-section of the bars are also used.

There are $n_{b}$ bars in the truss. The design variable is $s \in \mathbb{R}^{n_{b}}$ where $s_{i}$ is the cross-section area of bar $i$, which has length $l_{i}$. It is assumed that a likely state of the truss is unique and denoted $\left(\boldsymbol{u}(\boldsymbol{s}), \boldsymbol{p}_{n}(\boldsymbol{s}), \boldsymbol{p}_{o}(\boldsymbol{s}), \boldsymbol{p}_{t}(\boldsymbol{s})\right)$ for a given design $s$. (In practice it is probably enough if local uniqueness is at hand.) Using this notation the structural optimization problem is 


$$
\begin{array}{ll}
\min _{\boldsymbol{s}} & \max _{i \in\left[1 \ldots n_{c}\right]} p_{n, i}(\boldsymbol{s}) \\
\text { subject to } & \sum_{i=1}^{n_{b}} s_{i} l_{i} \leq V, \\
& \sum_{i=1}^{n_{c}} p_{n, i}(\boldsymbol{s}) \geq P, \\
& s_{i}^{1} \leq s_{i} \leq s_{i}^{\mathrm{u}}, \quad i=1 \ldots n_{b},
\end{array}
$$

where $0<s_{i}^{\mathrm{u}}$ and $s_{i}^{\mathrm{l}}$ are the upper and lower bounds on the cross-section areas, respectively. In sizing of a truss the stiffness matrix $\boldsymbol{K}$ depends linearly on the design variable, i.e.,

$$
\boldsymbol{K}(\boldsymbol{s})=\sum_{i=1}^{n_{b}} \boldsymbol{k}_{i} s_{i},
$$

where the $\boldsymbol{k}_{i}$ are constant matrices.

\section{Solving the structural optimization problem}

The structural optimization problem (18) is non-differentiable, i.e., there are designs for which the cost function is non-differentiable with respect to the design variable. This is partly due to the max-operation but, more importantly, because the functions $\left(\boldsymbol{u}, \boldsymbol{p}_{n}, \boldsymbol{p}_{o}, \boldsymbol{p}_{t}\right)$ may be non-differentiable with respect to the design variable. This is a profound theoretical difficulty, but in the present paper we make a simplistic assumption which seems to be satisfied in practice: we simply assume that non-differentiable points are never encountered during the optimization process. This assumption makes its possible to employ standard optimization algorithms for differentiable optimization problems to solve (18), even though one cannot expect any convergence proof to be valid. The assumption may be justified if the set of designs for which $\left(\boldsymbol{u}, \boldsymbol{p}_{n}, \boldsymbol{p}_{o}, \boldsymbol{p}_{t}\right)$ is non-differentiable is small compared to the size of the entire design-space.

We have chosen to use the method of moving asymptotes (MMA), see Svanberg (1987). The MMA was developed for differentiable optimization problems, but has been proven to work well also for non-differentiable problems of the present type, see Klarbring and Rönnqvist (1995). The MMA is similar to most standard sequential programming algorithms; to use the method it is necessary to be able to evaluate the cost function and all constraints for a given design as well as their derivatives with respect to the design variable. Earlier experience with the MMA for a similar problem with the same cost function as (18), Hilding et al. (1999), indicates 
that the efficiency of the MMA might be greatly improved if the following alternative form of (18) is used:

$$
\begin{aligned}
& \min _{\boldsymbol{s}} z \\
& \text { subject to } p_{n, i}(s) \leq z, i=1 \ldots n_{c} \text {, } \\
& \sum_{i=1}^{n_{b}} s_{i} l_{i} \leq V \\
& \sum_{i=1}^{n_{c}} p_{n, i}(s) \geq P \text {, } \\
& s_{i}^{1} \leq s_{i} \leq s_{i}^{\mathrm{u}}, i=1 \ldots n_{b} .
\end{aligned}
$$

Here, compared to (18), an additional variable $z$ has been introduced. The cost function and all constraints except the constraints involving $p_{n, i}$ are trivial to evaluate as they depend linearly on the design and the $z$ variable, and the same holds for their derivatives. Evaluating the functions $p_{n, i}$ in the remaining constraints requires solving the likely-state problem (8), which is done using the enumeration technique described in Hilding (2000). The derivatives of the latter constraints with respect to the design variable are calculated using the sensitivity analysis developed in Sec. 3.

\section{Numerical experiments}

In this section the results of some numerical experiments are presented. The structural optimization problem used is the truss-sizing problem defined in Sec. 4. The main assumption is that the solution of the likely-state problem (8) exists and is unique for all feasible designs $s$ of the structural optimization problem.

The following properties are shared by all numerical experiments below. The material used in the bars has a Young's modulus of $200 \mathrm{GPa}$. The upper limit on the cross-section areas is $0.1 \mathrm{~m}^{2}$ and the lower is $0.01 \mathrm{~m}^{2}$. In the initial design $s_{i}=0.02 \mathrm{~m}^{2}$ for all bars. The volume limit $V$ is set to the total volume of the bars in the initial design. The minimum total normal contact force $P$ is prescribed to be that of the initial design. The trusses are two-dimensional and to be able to use the theory in this paper, which is developed for three-dimensional trusses, the two-dimensional trusses are embedded in the $x-y$ plane and then all displacements in the $z$ direction are prescribed to be zero. In the numerical solution the alternative form (20) of (18) is used.

The optimization algorithm is implemented in MatLab. Running on an SUN Ultra 10 workstation (333 MHz CPU, SPECfp95 18.3), the time taken to optimize the trusses is less than 5 minutes. Implementing in a compiled 
language instead of in MatLab would increase the speed of the algorithm by possibly a factor of 10 to 100. A state-of-the-art CPU would obviously also make the computations considerably faster.

\subsection{Experiment 1}

The structural optimization problem (18) is solved for three truss-structures. These rectangular trusses are all of similar type, but have different number of nodes $(2 \times 5,3 \times 6$, and $4 \times 7)$, and since spacing between nodes are the same in all cases, their overall size differ. An example of such a truss is shown in Figure 3. For all three trusses it holds that they rest on

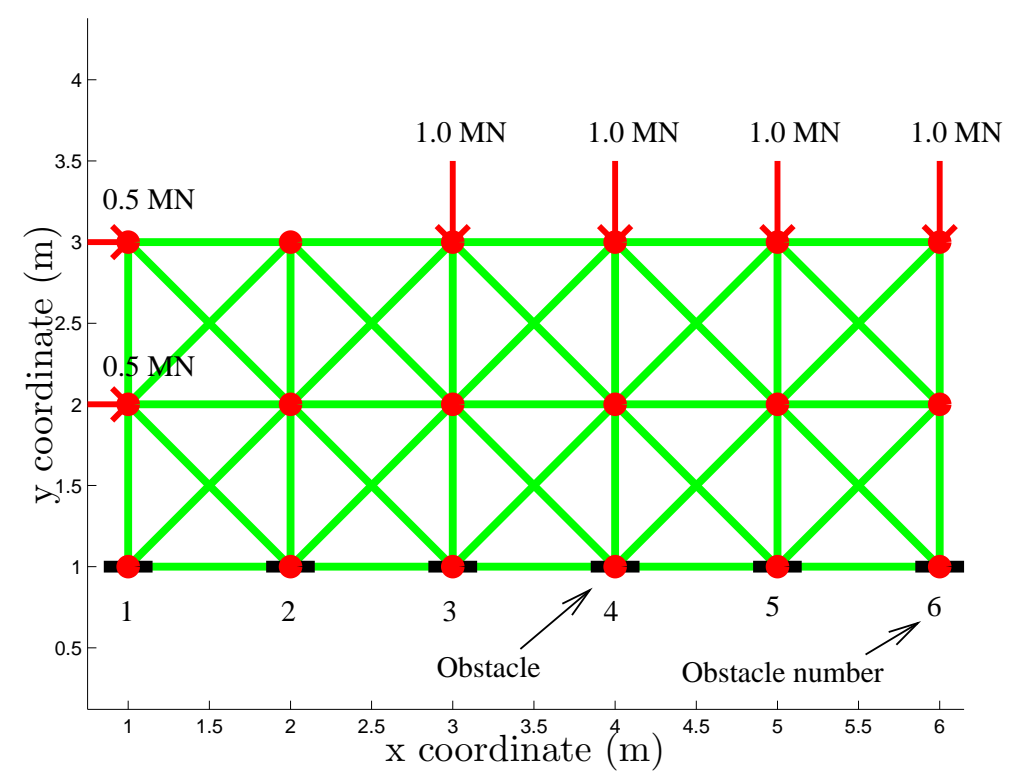

Figure 3: The truss to be optimized, in the case of $3 \times 6$ nodes.

a rigid plane with a friction coefficient of 0.5 , i.e., $\mu_{i}=0.5$, and the gap between structure and obstacle is zero, i.e., $\boldsymbol{g}=\mathbf{0}$. The static loads on the truss with $I \times J$ nodes are: (i) two loads of magnitude $0.5 \mathrm{MN}$ in the positive $x$ direction applied at nodes with $(x, y)$ coordinates: $(1, I) \mathrm{m}$ and $(1, I-1) \mathrm{m}$, and (ii) four loads of magnitude $1 \mathrm{MN}$ in the negative $y$ direction applied at nodes with coordinates $(J, I) \mathrm{m},(J-1, I) \mathrm{m},(J-2, I) \mathrm{m}$, and $(J-3, I) \mathrm{m}$. Note that due to the overall equilibrium, the sum of the normal contact forces must be $4 \mathrm{MN}$ for all designs. Hence, the total normal force constraint in (18) is really not necessary in this case.

The contact forces for the initial and optimized designs may be found in Figure 4. For all three trusses, it is clearly seen that the normal contact forces are more uniform in the optimized design than in the initial design. 

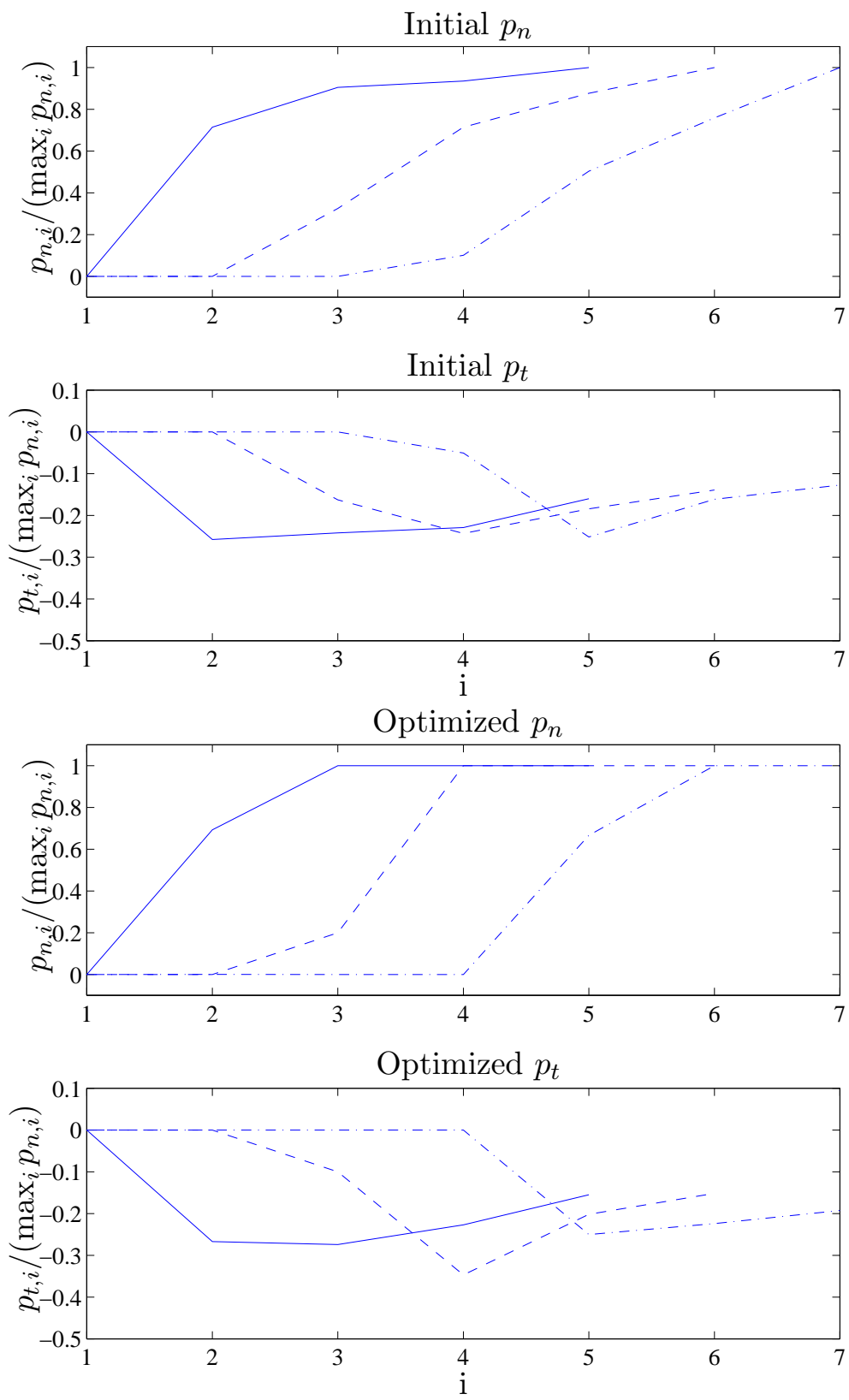

Figure 4: Contact forces of initial and optimized designs in Experiment 1. (Solid line: $2 \times 5$ truss, dashed line: $3 \times 4$ truss, and dash-dotted line: $4 \times 7$ truss.) 
The value of the cost function for the initial and optimized designs may be found in Table 1.

Table 1: Cost function values for initial and optimized designs for Experiment 1

\begin{tabular}{l|ll}
\hline Truss & $\max p_{n, i}$, initial design $(\mathrm{MN})$ & $\max p_{n, i}$, optimized design $(\mathrm{MN})$ \\
\hline $2 \times 5$ & 1.1257 & 1.0833 \\
$3 \times 6$ & 1.3718 & 1.2500 \\
$4 \times 7$ & 1.6929 & 1.5000
\end{tabular}

\subsubsection{On the quality of the obtained results in experiment 1}

The optimization problem (18) is a non-convex problem and one can in general not expect to find a globally optimal solution by a local search method as the one used here. However, a test for global optimum that may sometimes give a positive result is to formulate a convex relaxation of the original problem and then hope to show that this problem has the same optimal objective function value as the original problem. In fact, such a test works for the three trusses optimized in Experiment 1. Below, global optimum will be proven for the truss of size $2 \times 5$. The proofs for the two other trusses are analogous.

The force equilibrium (2) implies global equilibrium, i.e., balance of forces doing work in rigid body motions. Selecting the node with $(x, y)$ coordinates $(5,1) \mathrm{m}$ as moment point leads to the following equations for global equilibrium:

$$
\begin{aligned}
& p_{n, 1}+p_{n, 2}+p_{n, 3}+p_{n, 4}+p_{n, 5}=4 \mathrm{MN} \\
& p_{t, 1}+p_{t, 2}+p_{t, 3}+p_{t, 4}+p_{t, 5}=-1 \mathrm{MN} \\
& 4 p_{n, 1}+3 p_{n, 2}+2 p_{n, 3}+1 p_{n, 4}=5.5 \mathrm{MNm}
\end{aligned}
$$

Signorini's contact law (4) implies

$$
\boldsymbol{p}_{n} \geq \mathbf{0}
$$

The following relaxed optimization problem may now be created

$$
\begin{array}{ll}
\min _{\boldsymbol{p}_{n}, \boldsymbol{p}_{t}} & \max _{i \in[1 \ldots 5]} p_{n, i} \\
\text { subject to } & (21) \text { and }(22)
\end{array}
$$

It is straightforward to show that (23) has a solution and that the optimal value of the cost function is approximately $1.0833 \mathrm{MN}$. Furthermore, as only a subset of the constraints of (18) have been used in (23) and since 
they have the same optimal cost function value it follows that the optimal value of the optimization problem (23) must always be less than or equal to that of (18). Comparing the optimal value of (23) with that of (18), cf. Table 1, yields that they are the same. It follows that for the optimized design of truss $2 \times 5$ the value of the cost function is a low as possible for the problem (18). Hence, the optimized design is a global optimum of (18) and the proof is complete.

One may object that the above proof is not valid because the calculation and comparison of the optimal cost-function values of optimization problems (23) and (18) are not performed in exact arithmetic. However, an actual calculation showed that the relative difference is on the order of machine precision, approximately $10^{-16}$. Hence, this objection may not be of practical importance in this case.

One may also note that solving the optimization problem (23) obviously gives no information on the optimal design since this problem contains only contact forces as variables.

\subsection{Experiment 2}

The trusses used in this experiment are press-fitted, see Figure 5 for an illustration. The trusses are rectangular with sizes: $2 \times 4,3 \times 5$, and $4 \times 5$.

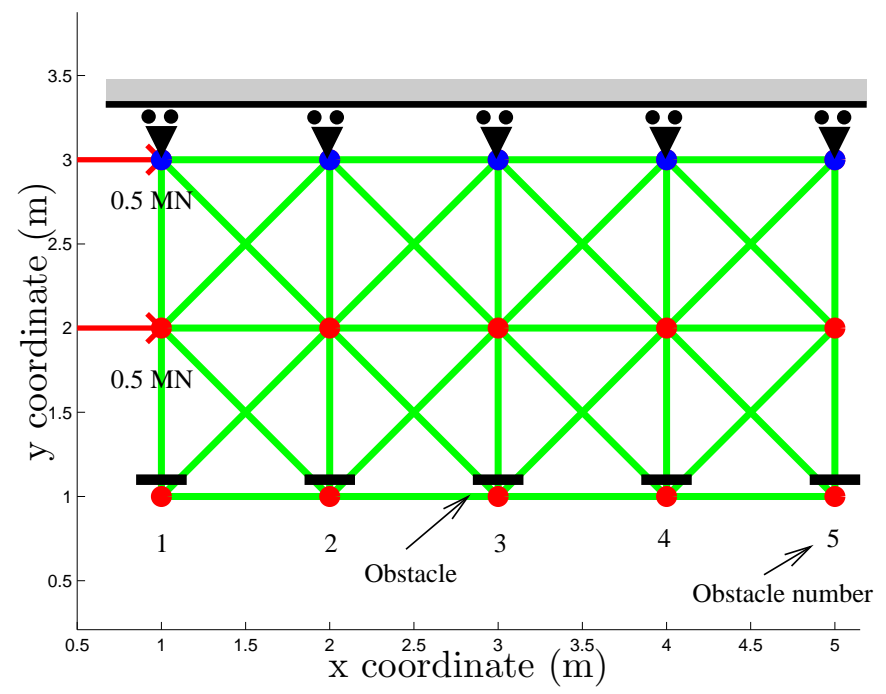

Figure 5: Truss of size $3 \times 5$.

The coefficient of friction is 0.5 , i.e., $\mu_{i}=0.5$, and the gap between structure and obstacle is $-0.001 \mathrm{~m}$, i.e., $g_{i}=-0.001 \mathrm{~m}$. The static loads on the truss 
of size $I \times J$ consists of two loads of magnitude $0.5 \mathrm{MN}$ in the positive $x$ direction applied at nodes with $(x, y)$ coordinates $(1, I) \mathrm{m}$ and $(1, I-1) \mathrm{m}$. The contact forces of the initial and optimized trusses are shown in Figure 6. It is clearly seen that the optimization method has succeeded in making the normal contact forces completely uniform, i.e., the optimized structures are globally optimal. The cost-function values of initial and optimized designs are shown in Table 2.

Table 2: Cost function values for initial and optimized designs for Experiment 2

\begin{tabular}{l|ll}
\hline Truss & $\max p_{n, i}$, initial design $(\mathrm{MN})$ & $\max p_{n, i}$, optimized design $(\mathrm{MN})$ \\
\hline $2 \times 4$ & 6.2648 & 5.6652 \\
$3 \times 5$ & 3.0674 & 2.8245 \\
$4 \times 6$ & 2.0440 & 1.8878
\end{tabular}

\section{Conclusions}

In this paper it is demonstrated that the likely-state problem can be used in optimization of structures in frictional contact subject to static loads. The sensitivity analysis of the likely-state problem, necessary to solve such structural optimization problems, is also developed. Although the developed theory and methods are demonstrated for truss-sizing problems, they are straightforward to use in sizing or shape optimization of finite element modeled structures.

The purpose of the numerical experiments in this paper is to illustrate the theory. Still, these small experiments indicate that the main computational effort lies in solving the likely-state problem for different designs. Hence, a more efficient solution technique for solving that problem than the one used in this paper would be valuable if structures with many contact constraints are to be optimized. 

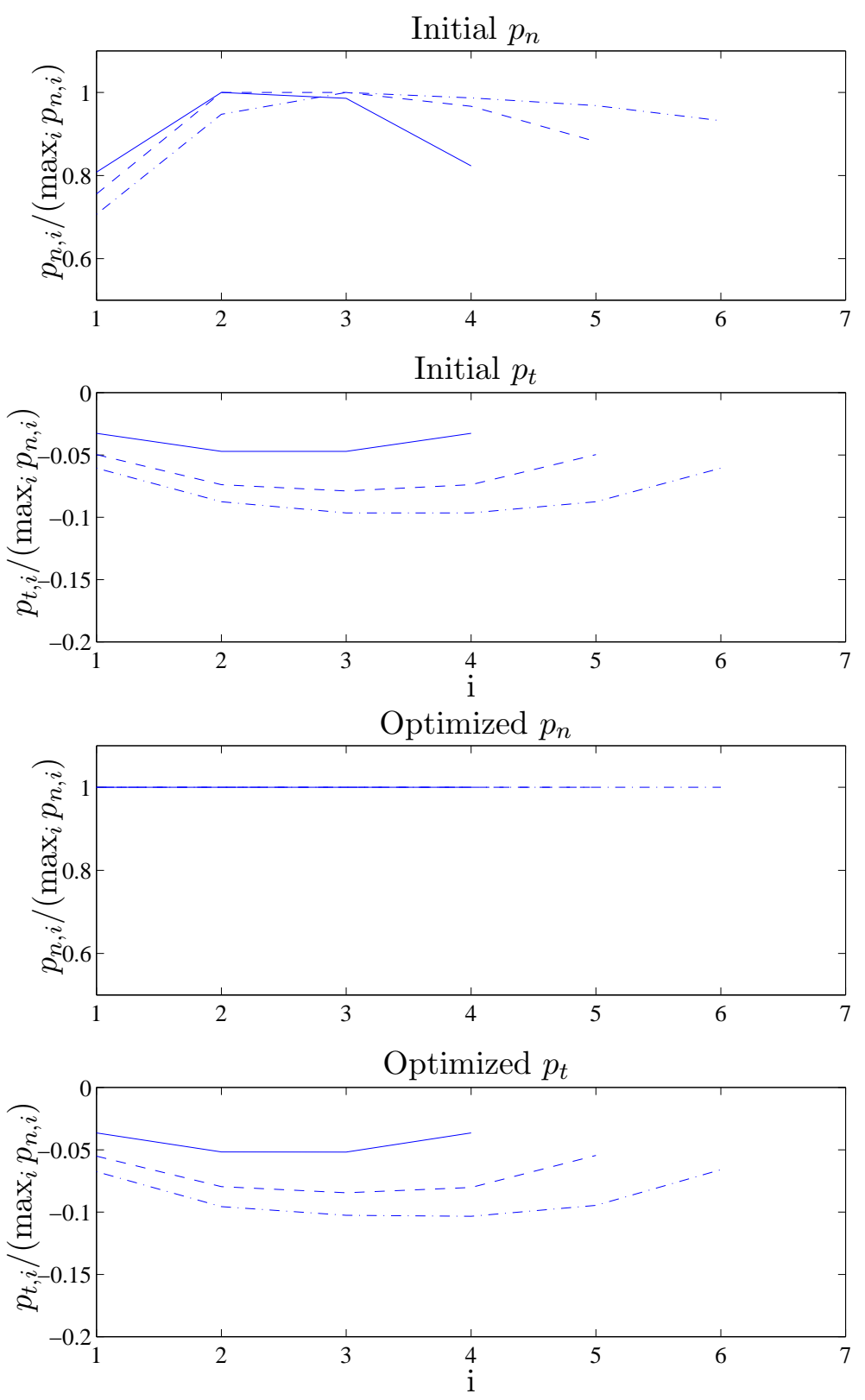

Figure 6: Contact forces of initial and optimized designs in Experiment 2. (Solid line: $2 \times 4$ truss, dashed line: $3 \times 5$ truss, and dash-dotted line: $4 \times 6$ truss.) 


\section{References}

Christensen, P. W. and Klarbring A. (2009). An introduction to structural optimization. Springer Science.

Duvaut, G. and Lions, J.-L. (1976). Inequalities in mechanics and physics. Springer-Verlag, Berlin. Translated from the French by C. W. John, Grundlehren der Mathematischen Wissenschaften, 219.

Fiacco, A. V. (1983). Introduction to sensitivity and stability analysis in nonlinear programming. Academic Press Inc., Orlando, Fla.

Golub, G. H. and van Loan, C. F. (1989). Matrix Computations. Johns Hopkins University Press, 2715 North Charles Street, Baltimore, Maryland 21218-4319. ISBN 0-8018-3772-1.

Haslinger, J. and Neittaanmaki, P. (1996). Finite element approximation for optimal shape, material and topology design. John Wiley \& Sons Ltd., Chichester, second edition.

Hilding, D. (2000). The equilibrium state of a structure subject to frictional contact. European Journal of Mechanics A/Solids, 19:1029-1040.

Hilding, D., Klarbring, A., and Pang, J.-S. (1999). Minimization of maximum unilateral force. Computer Methods in Applied Mechanics and Engineering, 177(3-4):215-234.

Klarbring, A., Mikelić, A., and Shillor, M. (1992a). Contact problems with friction and applications to shape optimization. In Theoretical aspects of industrial design (Wright-Patterson Air Force Base, OH, 1990), pages 83-91. SIAM, Philadelphia, PA.

Klarbring, A., Mikelić, A., and Shillor, M. (1992b). Optimal shape design in contact problems with normal compliance and friction. Appl. Math. Lett., 5(2):5155.

Klarbring, A. and Pang, J.-S. (1996). An MPEC approach to optimal design problems of discrete structures involving unilateral contact with Coulomb friction. In Abstracts of Euromech Colloquium 351: Systems with Coulomb friction, page 24. Euromech.

Klarbring, A. and Rönnqvist, M. (1995). Nested approach to structural optimization in nonsmooth mechanics. Structural Optimization, 10:79-86.

Kočvara, M. and Outrata, J. V. (1997). A nonsmooth approach to optimization problems with equilibrium constraints. In Complementarity and variational problems (Baltimore, MD, 1995), pages 148-164. SIAM, Philadelphia, PA.

Outrata, J., Kočvara, M., and Zowe, J. (1998). Nonsmooth approach to optimization problems with equilibrium constraints. Kluwer Academic Publishers, Dordrecht. Theory, applications and numerical results.

Rodrigues, H. C. (1993). A mixed variational formulation for shape optimization of solids with contact conditions. Structural Optimization, 6:19-28.

Stavroulakis, G. E. (1995). Optimal prestress of structures with frictional unilateral contact interfaces. Archive of Applied Mechanics, 66:71-81.

Svanberg, K. (1987). The method of moving asymptotes - a new method for structural optimization. International Journal for Numerical Methods in Engi- 
neering, 24:359-373. 